\title{
OPERATOR NORM AND NUMERICAL RADIUS ANALOGUES OF COHEN'S INEQUALITY
}

\section{ROMAN DRNOVŠEK}

Abstract. Let $D$ be an invertible multiplication operator on $L^{2}(X, \mu)$, and let $A$ be a bounded operator on $L^{2}(X, \mu)$. In this note we prove that $\|A\|^{2} \leqslant\|D A\|\left\|D^{-1} A\right\|$, where $\|\cdot\|$ denotes the operator norm. If, in addition, the operators $A$ and $D$ are positive, we also have $w(A)^{2} \leqslant$ $w(D A) w\left(D^{-1} A\right)$, where $w$ denotes the numerical radius.

Mathematics subject classification (2010): 47A30, 47A12, 47A10.

Keywords and phrases: Operator norm, numerical radius, spectral radius.

\section{REFERENCES}

[1] J. E. CohEn, Cauchy inequalities for the spectral radius of products of diagonal and nonnegative matrices, Proc. Amer. Math. Soc. 142 (2014), no. 11, 3665-3674.

[2] J. E. CoHEN, Stochastic population dynamics in a Markovian environment implies Taylor's power law of fluctuation scaling, Theoret. Population Biol. 93 (2014), 30-37.

[3] G. B. Folland, Real analysis. Modern techniques and their applications, Second edition, John Wiley \& Sons, New York, 1999.

[4] K. E. Gustafson, D. K. M. RaO, Numerical range. The field of values of linear operators and matrices, Universitext, Springer-Verlag, New York, 1997.

[5] Generalization of Holder's inequality, In Wikipedia. Retrieved January 31, 2019, from https://en.wikipedia.org/wiki/Hölder's inequality . 